\title{
Update on PCSK9 Inhibitors and New Therapies
}

\author{
Evan A Stein ${ }^{1}$ and Frederick J Raal² \\ 1. Metabolic and Atherosclerosis Research Center, Cincinnati, Ohio, US; 2. Carbohydrate and Lipid Metabolism Research Unit, Faculty of Health Sciences, \\ University of the Witwatersrand, Johannesburg, South Africa
}

\begin{abstract}
Proprotein convertase subtilisin/kexin type 9 (PCSK9), first described in 2003, binds to the low-density lipoprotein receptor (LDLR) resulting in its degradation. Inhibition of PCSK9 results in increased LDLR recycling and a reduction in LDL-cholesterol (LDL-C). The clinical development of monoclonal antibodies (mAbs) that bind to circulating PCSK9 has been rapid with large phase II and III trials demonstrating substantial reductions in LDL-C when given to a very broad group of patients including those with familial and non-familial hypercholesterolemia, diabetes, heart disease, and in those intolerant to statins. Despite sub-cutaneous administration these mAbs are well tolerated and have demonstrated good safety. Two agents, alirocumab and evolocumab, received regulatory approval in 2015 in the US and Europe and evolocumab in 2016 in Japan.
\end{abstract}

\section{Keywords}

PCSK9, low-density lipoprotein (LDL) cholesterol, LDL receptor, cardiovascular disease, familial hypercholesterolemia

Disclosure: Evan A Stein has received consulting fees from Amgen, Regeneron, Sanofi, Genentech, Roche, The Medicines Co, ISIS, Catabasis, AstraZeneca, CymaBay, CVS/ Caremark and BMS related to PCSK9 inhibitors and other lipid lowering drugs. Frederick J Raal has received honoraria and/or consulting fees from AstraZeneca, Pfizer, Merck, Amgen, Sanofi and his institution grants/research support from Amgen and Sanofi. This article is a short opinion piece and has not been submitted to external peer reviewers. No funding was received for the publication of this article.

Acknowledgments: The authors were solely responsible for the writing and submission of the manuscript.

Open Access: This article is published under the Creative Commons Attribution Noncommercial License, which permits any noncommercial use, distribution, adaptation, and reproduction provided the original author(s) and source are given appropriate credit.

Received: March 10, 2016 Published Online: April 15, 2016 Citation: US Endocrinology 2016;12(1):18-21 DOI: http://doi.org/10.17925/USE.2016.12.01.18

Correspondence: Evan A Stein, Director Emeritus, Metabolic and Atherosclerosis Research Center, 5355 Medpace Way, Cincinnati, OH, US. E: esteinmrl@aol.com

Statins, first approved for general use in 1987 based on their low-density lipoprotein cholesterol (LDL-C) lowering ability, took another decade to demonstrate cardiovascular disease (CVD) benefit. ${ }^{1-4}$ Since 1990 there have been more efficacious statins but only one other modestly effective LDL-C-reducing drug, ezetimibe, has been shown to significantly reduce CVD events compared to statin alone..$^{5-8}$

There remains an unmet need for patients, many at high CVD risk, who are unable to achieve 'optimal' LDL-C targets with statin therapy, or are intolerant to statins. Two new drugs were approved in 2013-2014 both of which inhibit production of $L D L$, or its precursor very $(V) L D L$; mipomersen, an apolipoprotein B antisense agent, and lomitapide, an inhibitor of microsomal triglyceride transport protein. ${ }^{9}$ However their use is strictly limited to the rare orphan population with homozygous familial hypercholesterolemia $(\mathrm{HoFH})$ with prescribing controlled by a Risk Evaluation and Monitoring Strategy in the USA and a 'named patient' program in other countries. ${ }^{9}$ Thus the proprotein convertase subtilisin/ kexin type 9 (PCSK9) monoclonal antibodies (mAbs), alirocumab and evolocumab, approved in late 2015, now provide a new class of drugs which substantially and safely decrease LDL-C. ${ }^{10-13}$

\section{Proprotein convertase subtilisin/kexin 9 inhibitors}

PCSK9 plays a key role in regulating LDL-C clearance by binding to the $\mathrm{LDL}$ receptor (LDLR) resulting in its degradation. ${ }^{14}$ Loss-of-function (LOF) mutations resulting in small lifelong LDL-C reductions are associated with a 40\% reduction in CVD risk. 15,16 In 2006, Legace discovered that circulating PCSK9 interacted with the LDLR providing a rationale for development of mAbs to inhibit PCSK $9 .{ }^{17}$ The first two PCSK9 mAbs, alirocumab and evolocumab, entered clinical trials in 2009 and have since demonstrated dramatic, rapid and persistent reductions in LDL-C in patients with a wide variety of lipid pheno- and genotypes, comorbidities and on a variety of background therapies. ${ }^{18-39}$ This resulted in both compounds receiving US Food and Drug Administration (FDA) and European Medicine's Agency (EMA) approval in late 2015. evolocumab received approval in Japan in early 2016.10-13,40 A third mAb, bococizumab, is still in phase III clinical development. ${ }^{41}$

\section{Pharmacokinetic and pharmacodynamics}

Following subcutaneous (SC) injection mAbs are rapidly absorbed into the circulation, bind to PCSK9, reducing free PCSK9 and reduce LDL-C within days. At a dose of $150 \mathrm{mg}$ LDL-C plateaus at roughly $60 \%$, which is maintained for two weeks. ${ }^{18,19} \mathrm{~A}$ three-fold increase to $420 \mathrm{mg}$ provides no additional LDL-C reduction but does suppress PCSK9 and LDL-C for four weeks. ${ }^{19}$

\section{Low-desity lipoprotein cholesterol in nonFH patients and influence of background therapy}

As statins cause an increase in synthesis and circulating PCSK9, it was suggested that inhibition of PCSK9 would be synergistic when added to 
Table 1: US Food and Drug Administration-approved indications, dosing and LDL-C reductions for Alirocumab (Praluent ${ }^{\circledR}$ ) and Evolocumab (Repatha ${ }^{\circledR}{ }^{21,22,43}$

$\begin{array}{ll} & \text { Alirocumab (Praluent }{ }^{\circledR} \text { ) } \\ \text { Indications } & \text { Primary hyperlipidemia: Indicated as adjunct to diet and } \\ & \text { maximally tolerated statin therapy for the treatment of adults } \\ & \text { with HeFH or clinical ASCVD, who require additional lowering } \\ & \text { of LDL-C }\end{array}$

Dosing Instructions

\begin{abstract}
Recommended starting dose is $75 \mathrm{mg}$ SC Q2W. If the LDL-C response is inadequate, the dosage may be increased to the maximum dosage of $150 \mathrm{mg}$ Q2W. Measure LDL-C levels within 4 to 8 weeks of initiating or titrating drug, to assess response and adjust the dose, if needed
\end{abstract}

\section{Evolocumab (Repatha ${ }^{\oplus}$ )}

Primary Hyperlipidemia: Indicated as an adjunct to diet and maximally tolerated statin therapy for treatment of adults with HeFH or clinical ASCVD who require additional lowering of LDL-C HoFH: Indicated as an adjunct to diet and other LDL-lowering therapies (e.g., statins, ezetimibe, LDL apheresis) in patients $\geq 13$ years with HoFH who require additional lowering of LDL-C.

Recommended dose in patients with $\mathrm{HeFH}$ or patients with primary hyperlipidemia with established clinical ASCVD is either SC 140 mg Q2W OR $420 \mathrm{mg}$ Q4W. For the $420 \mathrm{mg}$ dose, give 3 injections within 30 minutes. When switching dosage regimens, administer the first dose of the new regimen on the next scheduled date of the prior regimen. In HoFH the recommended dose is $420 \mathrm{mg}$ Q4W with LDL-C measured 4 to 8 weeks after starting, as response will depend on the degree of LDL-receptor function

140 mg Q2W or 420 mg Q4W: 60\% HoFH 420 mg Q4W: 31\%

Store refrigerated at $36^{\circ} \mathrm{F}$ to $46^{\circ} \mathrm{F}\left(2^{\circ} \mathrm{C}\right.$ to $\left.8^{\circ} \mathrm{C}\right)$ in the outer carton. Warm to room temperature for $\geq 30$ minutes.

The drug can also be kept at room temperature, up to $25^{\circ} \mathrm{C}\left(77^{\circ} \mathrm{F}\right)$ in the original carton but must be used within 30 days. statins. However, numerous trials performed in patients on diet alone, on existing statin therapy or randomized to various doses of statins have shown PCSK9 mAbs provide a consistent additional 50 to 60\% decrease in LDL-C. ${ }^{20-39}$ This implies that there may be a limit to the upregulation of LDLR activity at the higher doses of statins. Included in these trials have been a large number of elderly, diabetics, women, patients with CVD and other CVD risk factors making the results applicable to the likely population in which mAbs will be used. ${ }^{20-39}$

While mAbs were well tolerated in short-term eight- to 12-week phase II trials, concern remained regarding longer term adherence given that this was the first mainstream SC therapy for lowering LDL-C. ${ }^{20-22,29}$ This was addressed in phase III trials of 52 weeks or longer ${ }^{23-28,35,39}$ which all demonstrated adherence as good as trials with statins, ezetimibe and other oral lipid altering agents.

Three trials in patients unable to tolerate statins have been published or presented, two with evolocumab (GAUSS and GAUSS-2) and one with alirocumab (ODYSSEY Alternative). ${ }^{23,31,34}$ In these studies, the majority of patients were at high or very high risk for CVD and had mean baseline LDL-C of $>190 \mathrm{mg} / \mathrm{dL}$ emphasizing the significant unmet need for additional effective LDL-C lowering in these patients., In all three of these studies, despite significant reductions in LDL-C of 45 to $63 \%$, muscle adverse effects were uncommon and no more frequent than those seen with ezetimibe.

\section{Familial hypercholesterolemia}

In phase I studies in heterozygous $(\mathrm{He}) \mathrm{FH}$, both alirocumab and evolocumab demonstrated LDL-C reductions of similar magnitude to that seen in nonFH subjects. ${ }^{18,19}$ This was confirmed in larger phase $\|$ trials in $\mathrm{HeFH}$ patients already on maximal statin, with or without ezetimibe. ${ }^{21,30}$ Reductions in LDL-C were $60 \pm 5 \%$ with alirocumab $150 \mathrm{mg}$ once every two weeks (Q2W) and evolocumab 140 mg Q2W or 420 mg once every four weeks (Q4W). ${ }^{36}$ In the Rutherford-2 study mutation analysis causing $\mathrm{FH}$ was performed and showed similar LDL-C reductions irrespective of LDLR mutation. ${ }^{36}$ The global phase III alirocumab HeFH trials increased the number of patients and diversity of genetic mutations along with longer duration of therapy and confirmed that the reductions in LDL-C are maintained in the long term. ${ }^{24,28,39}$

Two trials with evolocumab in HoFH patients have been carried out; In TESLA part A, a small pilot proof-of-concept trial, 8 patients on maximal stable drug therapy were treated with evolocumab $420 \mathrm{mg}$ Q4W for 12 weeks. ${ }^{37}$ Six patients, with LDLR defective activity, experienced mean reductions in LDL-C of $19.3 \%$ while the two patients with LDLR negative activity had no response. ${ }^{37}$ The definitive phase III randomized, placebocontrolled, double-blind trial, TESLA part B, randomized 50 patients, 12 years or older on maximal statin \pm ezetimibe but not apheresis with mean baseline LDL-C of $348 \mathrm{mg} / \mathrm{dL} .{ }^{38}$ After 12 weeks LDL-C decreased by $31 \%$ compared to placebo, an absolute reduction in LDL-C of $93 \mathrm{mg} / \mathrm{dL}$. This trial confirmed that the response was dependent on residual LDLR activity, with a $41 \%$ reduction seen in those with at least one LDLR defective mutation and a $46 \%$ reduction seen in in those with two LDLR defective genes. ${ }^{38}$ Based on these results evolocumab was approved by FDA and EMA for treatment of HoFH. ${ }^{11,12}$

\section{Effect on lipoprotein(a) and other lipids and lipoproteins}

As expected PCSK9 mAbs reduce apolipoprotein B and related lipids and lipoproteins in parallel to LDL-C, along with small reductions in triglycerides and increases in high-density lipoprotein (HDL)-C. ${ }^{42}$ An unexpected effect has been a robust and consistent reduction in lipoprotein(a) (Lp[a]) of 25\% to $30 \%$ seen with both alirocumab and evolocumab. ${ }^{42}$ 


\section{Safety, tolerability and cardiovascular Events}

No specific or serious clinical or laboratory adverse events have been identified in the $>10,000$ patients who have been treated with PCSK9 mAbs for durations of 3+ years. ${ }^{20-39}$ Despite the need for SC injections, they have been well tolerated with few injection site reactions..$^{20-39}$ No physical, psychological, endocrine or reproductive abnormalities or 'off-target' effects have been observed in these large trials. Careful examination, by the drug sponsors, and FDA regulators, of patients achieving very low LDL-C levels ( $<25$ or $<15 \mathrm{mg} / \mathrm{dL}$ ) found no differences in adverse events compared to those with higher LDL-C, or those treated with placebo or standard of care. ${ }^{43}$ However, as with statins, it may take decades of therapy in many thousands of patients to detect more subtle side effects not readily apparent in relatively short term trials.

A recently published predefined exploratory and a post hoc analysis assessing CVD events from two phase III trials with evolocumab and alirocumab respectively showed very similar 50\% reductions in CVD events compared to standard of care or placebo. ${ }^{34,45}$ Although the number of events were small, both studies were statistically significant. While these early results are encouraging, definitive CVD outcome trials are already underway and will also address long term safety concerns. ${ }^{49-51}$ Results from the 27,500-patient Further Cardiovascular Outcomes Research With PCSK9 Inhibition in Subjects With Elevated Risk (FOURIER
NCT01764633), trial comparing evolocumab 150 mg Q2W or 420 mg Q4W added to statin versus statin alone, are expected in late 2016.28,39

\section{Approved indications and annual Cost}

In the USA the approved indications (Table 1) are slightly more restrictive than that approved in Europe; both drugs are approved for use in adults with $\mathrm{HeFH}$ or clinical atherosclerotic CVD, on maximally tolerated statin therapy who require additional LDL-C lowering. In addition evolocumab is approved for $\mathrm{HoFH}$ patients aged 13 or older. ${ }^{10,11}$ The reported list price for alirocumab, $75 \mathrm{mg}$ or $150 \mathrm{mg}$ doses is $\$ 14,600$ per year, and for evolocumab $140 \mathrm{mg}$ or $420 \mathrm{mg} \$ 14,100.44,45$ Actual costs to health insurance payers will likely be significantly less. Thus it is important that they be used only where appropriate, after implementation of maximal tolerable doses of the most efficacious statins, atorvastatin and rosuvastatin, plus ezetimibe.

\section{Conclusion}

The PCSK9 inhibitors have shown excellent LDL-C lowering efficacy, good safety and encouraging early CVD results resulting in regulatory approval of a new therapeutic class of drugs which have the potential to surpass the impact of statins. Large CVD outcome trials are due to be completed starting in 2016 and will likely provide impetus for more widespread use of these agents resulting in further reductions in the burden of CVD. ${ }^{46,47}$
1. Tobert JA, Lovastatin and Beyond: The History of the HMG COA Reductase Inhibitors, Nat Rev Drug Discov, 2003:2:517-526.

2. Pedersen TR, Kjekshus J, Berg K, et al., Scandinavian Simvastatin Survival Study Group. Randomised trial of cholesterol lowering in 4444 patients with coronary heart disease: the Scandinavian Simvastatin Survival Study (4S), Lancet, 1994:344:1383-1389.

3. Shepherd J, Cobbe SM, Ford I, et al., for the West of Scotland Coronary Prevention Study Group. Prevention of coronary heart disease with pravastatin in men with hypercholesterolemia, N Eng/ J Med, 1995;333:1301-1307.

4. Sacks FM, Pfeffer MA, Moye LA, et al., for the Cholesterol and Recurrent Events Trial Investigators. The effect of pravastatin on coronary events after myocardial infarction in patients with average cholesterol levels, N Eng/ I Med, 1996:335:1001-1009.

5. Heinonen T, Stein EA, Weiss S, et al., The Lipid-Lowering Effects of Atorvastatin, a New HMG-COA Reductase Inhibitor: Results of a Randomized, Double-Masked Study, Clinical Therapeutics, 1996;18:853-863.

6. Jones PH, Davidson MH, Stein EA, et al., Comparison of the Efficacy and Safety of Rosuvastatin Versus Atorvastatin Simvastatin, and Pravastatin Across Doses (Stellar* Trial), Am J Cardiol, 2003:92:152-160.

7. Stein EA, Results of Phase I/II Clincial Trials with Ezetimibe, a Novel Selective cholesterol Absorption Inhibitor, Euro Heart Journal, 2001;3E;11-16.

8. Cannon CP, Blazing MA, Giugliano RP, et al., Ezetimibe Added to Statin Therapy after Acute Coronary Syndromes, N Eng/ J Med, 2015; 372:2387-97

9. Rader DJ, Kastelein JJ, Lomitapide and mipomersen: two firstin-class drugs for reducing low-density lipoprotein cholesterol in patients with homozygous familial hypercholesterolemia, Circulation, 2014; 129: 1022-32.

10. FDA approves Praluent to treat certain patients with high cholesterol. Available at: http://www.fda.gov/NewsEvents/ Newsroom/PressAnnouncements/ucm455883.htm. (accessed February 28, 2016)

11. FDA approves Repatha to treat certain patients with high cholesterol. Available at: http://www.fda.gov/NewsEvents/ Newsroom/PressAnnouncements/ucm460082.htm. (accessed February 28, 2016)

12. Repatha/evolocumab. Available at: http://www.ema.europa.eu/ docs/en_GB/document_library/Summary_of_opinion___Initial_ authorisation/human/003766/WC500187093.pdf. (accessed February 28, 2016)

13. Praluent recommended for approval to lower cholesterol. Available at: http://www.ema.europa.eu/docs/en_GB/ document_library/Press_release/2015/07/WC500190458.pdf (accessed February 28, 2016).

14. Turner T, Raal FJ, Stein EA, Evolving Targets of Therapy: Proprotein Convertase Subtilisin/Kexin 9 Inhibition. In: Ballantyne C, ed. Clinical Lipidology: A Companion to Braunwald's Heart Disease. 2nd ed: Elsevier; 2014:346-57.

15. Cohen JC, Boerwinkle E, Mosley TH Jr, Hobbs HH, Sequence variations in PCSK9, loW LDL, and protection against coronary heart disease, N Engl J Med, 2006;354:1264-72.

16. Kathiresan $S$ and the Myocardial Infarction Genetics Consortium, A PCSK9 Missense Variant Associated with a Reduced Risk of Early-Onset Myocardial Infarction, N Eng/ J Med, 2008:358:2299-2300.

17. Lagace TA, Curtis DE, Garuti R, et al., Secreted PCSK9 decreases the number of LDL receptors in hepatocytes and in livers of parabiotic mice, I Clin Invest, 2006;116:2995-3005.

18. Stein EA, Mellis $S$, Yancopoulos GD, et al., Effect of a Monoclonal Antibody to PCSK9 on LDL Cholesterol, N Eng/ J Med, 2012;366:1108-18.

19. Dias CS, Shaywitz AJ, Wasserman SM, et al., Effects of AMG 145 on low-density lipoprotein cholesterol levels: results from 2 randomized, double-blind, placebo-controlled, ascending-dose phase 1 studies in healthy volunteers and hypercholesterolem subjects on statins. J Am Coll Cardiol, 2012;60:1888-98.

20. Roth EM, McKenney JM, Hanotin C, et al., Atorvastatin with or without an antibody to PCSK9 in primary hypercholesterolemia, N Eng/ J Med, 2012;367:1891-900.

21. Stein EA, Gipe $D$, Bergeron J, et al., Effect of a monoclonal antibody to PCSK9, REGN727/SAR236553, to reduce low-density lipoprotein cholesterol in patients with heterozygous familial hypercholesterolaemia on stable statin dose with or withou ezetimibe therapy: a phase 2 randomised controlled trial, Lancet, 2012;380:29-36.

22. McKenney JM, Koren MJ, Kereiakes DJ, et al., Safety and efficacy of a monoclonal antibody to proprotein convertase subtilisin/kexin type 9 serine protease, SAR236553/REGN727, in patients with primary hypercholesterolemia receiving ongoing stable atorvastatin therapy, J Am Coll Cardiol, ongoing stable at

23. Moriarty PM, Jacobson TA, Bruckert E, et al., Efficacy and safety of alirocumab, a monoclonal antibody to PCSK9, in statin-intolerant patients: design and rationale of ODYSSEY ALTERNATIVE, a randomized phase 3 trial, I Clin Lipidol, 2014:8:554-61.

24. Kastelein JJP, Ginsberg HN, Langslet G, et al. ODYSSEY FHI and FH II: 78 week results with alirocumab treatment in 735 patients with heterozygous familial hypercholesterolaemia, Eur Heart J, 2015;36:2996-3003

25. Cannon CP, Cariou P, Blom D, et al., Efficacy and safety of alirocumab in high cardiovascular risk patients with inadequately controlled hypercholesterolaemia on maximally tolerated doses of statins: the ODYSSEY COMBO II randomized controlled trial, Eur Heart 1, 2015: 36:1186-94

26. Bays $\mathrm{H}$, Gaudet $\mathrm{D}$, Weiss $\mathrm{R}$, et al., Alirocumab as Add-On to Atorvastatin Versus Other Lipid Treatment Strategies: ODYSSEY OPTIONS I Randomized Trial, I Clin Endocrinol Metab, 2015;100:3140-8

27. Farnier M, Jones P, Severance R, et al., Efficacy and safety of adding alirocumab to rosuvastatin versus adding ezetimibe or doubling the rosuvastatin dose in high cardiovascular- risk patients: The ODYSSEY OPTIONS II randomized trial, Atherosclerosis, 2016:244:138-46.

28. Robinson JG, Farnier M, Krempf M, et al.. Efficacy and Safety of Alirocumab in Reducing Lipids and Cardiovascular Events, N Eng/ J Med, 2015: 372:1489-1499.

29. Stein EA, Giugliano RP, Koren MJ, et al., Efficacy and safety of evolocumab (AMG 145), a fully human monoclonal antibody to PCSK9, in hyperlipidaemic patients on various background lipid therapies: pooled analysis of 1359 patients in four phase 2 trials, Eur Heart J, 2014;35:2249-59.

30. Raal F, Scott R, Somaratne R, et al., Low-density lipoprotein cholesterol-lowering effects of AMG 145, a monoclonal antibody to proprotein convertase subtilisin/kexin type 9 serine protease in patients with heterozygous familial hypercholesterolemia: the Reduction of LDL-C with PCSK9 Inhibition in Heterozygous Familial Hypercholesterolemia Disorder (RUTHERFORD) randomized trial, Circulation, 2012;126:2408-17.

31. Sullivan $D$, Olsson $A G$, Scott $R$, et al., Effect of a monoclonal antibody to PCSK9 on low-density lipoprotein cholesterol levels in statin-intolerant patients: the GAUSS randomized trial, JAMA, 2012:308:2497-506.

32. 32. Giugliano RP, Desai NR, Kohli P, et al., Efficacy, safety, and tolerability of a monoclonal antibody to proprotein convertase subtilisin/kexin type 9 in combination with a statin in patients with hypercholesterolaemia (LAPLACE-TIMI 57): a randomised, placebo-controlled, dose-ranging, phase 2 study, Lancet, 2012;380:2007-17.

33. Koren MJ, Scott R, Kim JB, et al., Efficacy, safety, and tolerability of a monoclonal antibody to proprotein convertase subtilisin/kexin type 9 as monotherapy in patients with hypercholesterolaemia (MENDEL): a randomised, double-blind, placebo-controlled, phase 2 study, Lancet, 2012;380:1995-2006

34. Stroes E, Colquhoun D, Sullivan D, et al., Anti-PCSK9 Antibody Effectively Lowers Cholesterol in Patients With Statin Intolerance. The GAUSS-2 Randomized, Placebo-Controlled Phase 3 Clinical Trial of Evolocumab, J Am Coll Cardiol, 2014:63:2541-8.

35. Blom DJ, Hala T, Bolognese M, et al., A 52-Week PlaceboControlled Trial of Evolocumab in Hyperlipidemia, N Engl I Med 2014;370:1809-19.

36. Raal FJ, Stein EA, Dufour R, et al, for the RUTHERFORD-2 Investigators. PCSK9 inhibition with evolocumab (AMG 145) in heterozygous familial hypercholesterolaemia (RUTHERFORD-2): a randomised, double-blind, placebo-controlled trial, Lancet, 2015:385:331-340.

37. Stein EA, Honarpour $N$, Wasserman SM, et al. Effect of the Proprotein Convertase Subtilisin/Kexin 9 Monoclonal Antibody, AMG 145, in Homozygous Familial Hypercholesterolemia, Circulation, 2013;128:2113-2120.

38. Raal FJ, Honarpour N, Blom DJ, et al., Inhibition of PCSK9 with evolocumab in homozygous familial hypercholesterolaemia (TESLA Part B): a randomised, double-blind, placebo-controlled trial, Lancet, 2015;385:341-50. 
39. Sabatine MS, Giugliano RP, Wiviott SD, et al., on behalf of the OSLER Investigators. Efficacy and Safety of Evolocumab in Reducing Lipids and Cardiovascular Events, New Eng/ I Med, 2015;372:1500-9.

40. Amgen's Repatha ${ }^{\circledast}$ (Evolocumab) Approved In Japan. Available at: http://www.prnewswire.com/news-releases/amgensepatha-evolocumab-approved-as-irst-pcsk9-inhibitor-inapan-for-the-treatment-of-high-cholesterol-300208360.htm . (accessed February 28, 2016).

41. Ballantyne CM, Neutel J, Cropp A, et al., Results of bococizumab, a monoclonal antibody against proprotein convertase subtilisin/kexin type 9 , from a randomized, placebo- controlled, dose-ranging study in statin-treated subjects with hypercholesterolemia, Am J Cardiol, 2015:115:1212-21.

42. Stein EA, Raal FJ, Reduction of Low Density Lipoprotein Cholesterol by Monoclonal Antibody Inhibition of PCSK9, Ann Rev Medicine, 2014;65:417-31

43. Alirocumab Briefing Document: Endocrine \& Metabolic Advisory Committee Meeting, June 9 2015. Available at: http://www.fda.gov/downloads/ AdvisoryCommittees/CommitteesMeetingMaterials/Drugs/ EndocrinologicandMetabolicDrugsAdvisoryCommittee/ UCM449865.pdf. (accessed February 28, 2016).

44. Evolocumab (Repatha) - Another PCSK9 Inhibitor to Lower LDL-
Cholesterol, Med Lett Drugs Ther, 2015; 57(1479):140

45. Alirocumab (Praluent) to lower LDL-cholesterol, Med Lett Drugs Ther, 2015; 57:113.

46. Husten L CardioBrief First PCSK9 Drug Outcomes Trial Due in 2016 - FOURIER set to finish ahead of schedule, Medpage Today Dec. 22, 2015. Available at: http://www.medpagetoday.com/ Cardiology/CardioBrief/55379. (accessed December 30, 2016 ). 47. Schwartz GG, Bessac $L$, Berdan $L G$, et al., Effect of alirocumab, a monoclonal antibody to PCSK9, on long-term cardiovascular outcomes following acute coronary syndromes: Rationale and design of the ODYSSEY Outcomes trial, Am Heart J,

2014;168:682-689. 